\title{
Resolution recovery in Turbo Spin Echo using segmented Half Fourier acquisition
}

\author{
Cristina Santa-Marta ${ }^{\mathrm{a}, \mathrm{b}}$, Javier Lafuente ${ }^{\mathrm{a}}$, Juan José Vaquero ${ }^{\mathrm{a}}$, Pedro Garcia-Barreno ${ }^{\mathrm{a}}$, \\ Manuel Desco ${ }^{\mathrm{a}, *}$ \\ aUnidad de Medicina y Cirugla Experimental, Hospital General Universitario “Gregorio Maranon,” Madrid, Spain \\ ${ }^{\mathrm{B}}$ Dept. Fisica Matematica y Fluidos, UNED, Madrid, Spain
}

\begin{abstract}
Turbo Spin Echo (TSE) is a sequence of choice for obtaining $\mathrm{T}_{2}$-weighted images. TSE reduces acquisition time by acquiring several echoes within each TR, at the cost of introducing an exponential weighting in the $k$-space that leads to a certain image blurring. This is particularly important for short- $\mathrm{T}_{2}$ structures, which can even disappear if their size in the phase encoding direction is comparable to the degree of blurring. This article suggests the use of a combination of Half Fourier (HF) and segmented (multishot) TSE (sHF-TSE) to recover the original resolution of the SE images. The improved symmetry of the dataset achieved by HF reconstruction is used to increase the resolution of the TSE images. The proposed combination, available in most clinical scanners, reduces the blurring artifact inherent to the TSE sequence without increasing the scan time or the number of acquisitions, but at the cost of a slight reduction of the signal-to-noise ratios (SNR). Qualitative and quantitative results are presented using both numerical simulation and imaging. Significan edge enhancement has been achieved for structures with short $\mathrm{T}_{2}$, (narrowing of the full width at half maximum [FWHM] up to 45\%). The proposed sequence is more sensitive to movement artifacts but has proven to be superior to the conventional TSE for imaging static structures. C 2004 Elsevier Inc.
\end{abstract}

Keywords TSE; FSE; Resolution; SNR; $k$-Space

\section{Introduction}

The most widely used clinical magnetic resonance imaging techniques for the diagnosis of parenchymal disease employ heavily $T_{2}$-weighted sequences to detect long $T_{2}$ components in tissue. But tissues also contain short $T_{2}$ components that are not detected or only poorly detected with conventional sequences. These components are the majority species in tendons, ligaments, menisci and other related tissues, and the minority in many other tissues that have predominantly long $T_{2}$ components [1]. In this work we propose to use the properties of the segmented Half Fourier Turbo Spin Echo (sHF-TSE) sequence as a strategy to increase the spatial resolution of $\mathrm{T}_{2}$ weighted images and, in particular, the conspicuity of short $\mathrm{T}_{2}$ structures.

Turbo or Fast Spin Echo (TSE, FSE) [2], formerly
RARE (Rapid Acquisition with Relaxation Enhancement), is a faster alternative to the classical spin echo (SE) sequence [3] proposed by Hennig et al. [4] for the acquisition of $\mathrm{T}_{2}$-weighted images. The TSE sequence shortens the acquisition time by fillin in several lines of the $k$-space in each shot or repetition time (TR). The number of echoes ( $k$-space lines) acquired within each TR is called echo train length (ETL) and typically ranges from 3 to 30 [5]. The $k$-space thus becomes divided into segments, each one containing the echoes acquired at the same TE [6,7]. The number of segments corresponds to the number of echoes in the ETL.

The particular phase encoding strategy followed in TSE can significantl influenc the quality of the images, since the echo amplitude is modulated as a function of its position in the $k$-space: each segment becomes weighted according to the TE of their constituent echoes. The effects of this modulation in TSE imaging are ghosting and blurring artifacts that lead to a resolution loss in the phase encoding direction. These problems are particularly severe for 
short- $\mathrm{T}_{2}$ small objects that span only a few pixels in the phase encoding direction imaged using long echo train lengths; these structures may even disappear if the image is acquired using a long enough ETL [8].

Several phase encoding strategies for TSE imaging have been proposed to minimize these undesired effects $[9,10]$, obtaining either edge-enhanced images, when high spatial frequencies are collected at the early echoes, or blurred images with reduced truncation artifact, when the high spatial frequencies are collected at late echoes.

An alternative approach suggested to reduce the effects of the $\mathrm{T}_{2}$ modulation of the $k$-space is the use of postprocessing techniques [11]. Most of these procedures, however, require additional data acquisitions to average the signal decay for the echo train, and the resulting images show lower signal-to-noise ratios (SNR) than the original ones. Another method proposed to reduce the modulation of the echo amplitudes is the use of a small $\mathrm{f}$ ip angle refocusing pulse [12]. This technique generates a series of echoes that represent a complex combination of spin echoes and stimulated echoes. The resulting images show mixed $\mathrm{T}_{1}-\mathrm{T}_{2}$ contrast and also lower SNR than the images acquired by the standard TSE technique.

This work proposes to exploit the properties of the sHFTSE sequence to reduce the effect of the $k$-space modulation in TSE images. The sHF-TSE sequence is a Turbo Spin Echo sequence in which the f nal echoes of each echo train are calculated from the initial ones instead of acquired. This sequence increases the symmetry of the echo train, compensating for the $T_{2}$ decay at the second half of the echo train, and giving rise to images with higher resolution. Segmented Half Fourier imaging can be implemented with routine software in most clinical scanners, which makes this strategy easy to be adopted.

This work includes a systematic comparative study between the sHF-TSE and its equivalent $\mathrm{T}_{2}$-weighted TSE sequence, based both on numerical simulation and MRI datasets obtained from both phantoms and patients.

\section{Material and methods}

\subsection{Segmented Half Fourier in Turbo Spin Echo: theoretical basis}

Half Fourier (HF) or partial Fourier imaging is a reconstruction method that allows the reduction of imaging time by up to almost $50 \%$. Using HF technique, the entire MR image is reconstructed from as few as one-half of the number of phase-encoding steps. The lacking $k$-space data are calculated as the symmetric complex conjugate of each element with respect to the origin (center) of the matrix (Hermitian symmetry). In practice, image data sets always contain some phase errors that prevent a perfect conjugate symmetry of the $k$-space [13]. As implemented in clinical systems, HF imaging requires sampling of slightly over half the lines. The extra lines are used to generate phase correction maps that allow for a more accurate prediction of the missing values.

Half Fourier technique yields lower SNR than a standard full acquisition. The total signal amplitude in a full acquisition sequence is proportional to the number of samples $\mathrm{N}_{\mathrm{x}} \mathrm{N}_{\mathrm{y}}$, and the total (uncorrelated) noise results from adding noise powers, thus being proportional to $\left(\mathrm{N}_{\mathrm{x}} \mathrm{N}_{\mathrm{y}}\right)^{1 / 2}$. In HF sequences, noise becomes partially correlated and the total noise must be calculated as sum of powers for the uncorrelated points and sum of amplitudes for the correlated ones. The SNR loss with respect to the full acquisition [14] is given by:

$$
\frac{H F_{\text {_noise }}}{\text { fullacq_noise }}=\left(\frac{4^{*} p C+(p A-p C)}{100}\right)^{1 / 2}
$$

being $\mathrm{pA}$ the percentage of the dataset actually acquired (50 $\leq \mathrm{pA} \leq 100)$ and $\mathrm{pC}$ the percentage of calculated data ( $\mathrm{pC}$ $=100-\mathrm{pA}) \cdot \mathrm{pA}-\mathrm{pC}$ and $2 \mathrm{pC}$ thus represent the percentage of uncorrelated and correlated data in the $k$-space. A common practice is to acquire $60 \%$ of the lines and to calculate the remaining $40 \%$. In this case, the SNR decreases by a factor of 1.34 with respect to the full acquisition.

The echo train length (ETL) of the TSE sequence (parameter that determines the reduction of acquisition time in comparison with the SE sequence) requires a redef nition when dealing with sHF-TSE sequences: in conventional TSE sequences the number of segments of the $k$-space matches the number of echoes collected in each TR, but this no longer holds for sHF-TSE. Therefore, two different parameters are required to characterize the echo train:

1. ETL: the number of echoes actually acquired after the RF excitation pulse.

2. $\mathrm{ETL}_{\text {eff }}$ (effective ETL): the number of segments in the $k$-space, that is, the echo train length of a corresponding full acquisition sequence.

For an sHF-TSE sequence the relation between them is given by:

$$
\mathrm{ETL}=\text { integer }\left(\mathrm{ETL}_{\mathrm{eff}} * \mathrm{pA}\right) / 100
$$

The interesting feature of the SHF-TSE sequence is that it improves the symmetry of the echo train, since the calculated echoes at the end of the $k$-space have the same amplitude as the initial ones (Fig. 1). This is particularly signif cant for long echo trains (heavily $\mathrm{T}_{2}$-weighted images). The expected outcome of this increased symmetry is an improvement of the spatial resolution.

\section{Evaluation}

The sHF-TSE sequence has been characterized by measuring the signal level and blurring on three types of images: software phantoms, true images of physical phantoms, and patient images. 


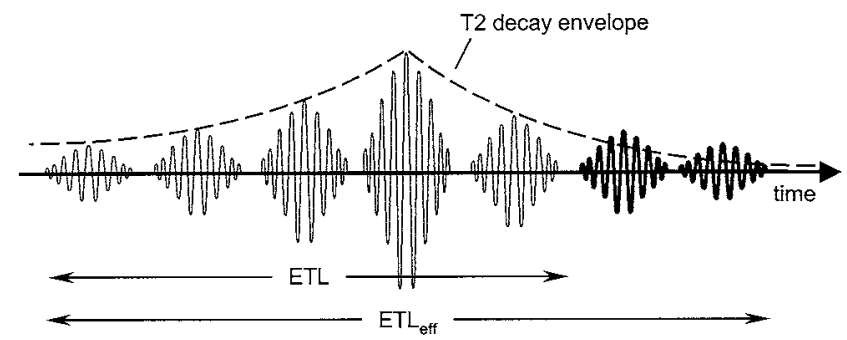

Fig. 1. Echo train for an sHF-TSE acquisition with ETL $=5$ and $\mathrm{ETL}_{\mathrm{eff}}=$ 7. The two f nal (bold) echoes are the calculated ones; note that if they were truly acquired their amplitudes should had decreased by the $T_{2}$ decay, represented by the dashed line.

Software phantom images allow the study of the effects derived purely from the phase encoding strategy, excluding any other inf uence of the imaging system. In particular, a one-pixel-line phantom (delta phantom) provides the point spread function (PSF) ${ }^{1}$ of the sequence. Height and full width at half maximum (FWHM) of the PSF, for a given $\mathrm{T}_{2}$, are direct measurements of the signal level and blurring of the image [16], respectively. These images were generated by means of computer simulation (described below).

Images of physical phantoms are also useful to assess the artifacts produced by the sequence in the presence of sharpedged objects. In this study, a physical phantom similar to one of the synthetic phantoms has been used both to validate the results of the simulation and to assess the artifacts introduced by the scanner by measuring blurring, signal level, and ringing on the true images.

Finally, we evaluated the real performance of the sequence using patient images from two different anatomical locations: joints and abdomen; these images allowed the assessment of blurring, signal level, and motion artifacts arising from soft edged biological structures.

\subsection{Synthetic images and computer simulation}

Two synthetic phantoms were designed: a "delta phantom" to measure the effective PSF of the sHF-TSE sequence, and a "three-circles phantom" to assess image blurring and ringing.

The delta phantom consists of a one-pixel width horizontal line (perpendicular to the phase encoding direction) in the center of the image $(y=0)$. The line intensity can be adjusted to simulate different $T_{2} s$. The range of $T 2$ values studied includes those typically found in clinical MR imaging: $\mathrm{T}_{2}=30,62$ and $126 \mathrm{~ms}$, roughly equivalent to those referred in the literature for muscle, fat, and tumor, respectively $[17,18]$. Longer $T_{2}$ values are much less affected by

\footnotetext{
${ }^{1}$ The concept of PSF cannot be strictly applied to TSE sequences since it depends on the object $T_{2}$. Therefore, to be formally correct, it should be called "effective PSF" and always referred to a particular $T_{2}$ [15] In this paper the term PSF always means PSF $_{\text {eff. }}$.
}

blurring and have not been considered in the experiment. The main parameters of the series of sequences simulated for this phantom are: $\mathrm{TR}=2500 \mathrm{~ms}, \mathrm{TE}=120 \mathrm{~ms}, \mathrm{pA}=$ $100,90,80,70$ and $60 \%$ and $\mathrm{ETL}_{\text {eff }}=256,21,15$ and 9 echoes [19]. TR and $\mathrm{TE}_{\text {eff }}$ were kept constant along the simulation so as to guarantee that image contrast depended only on the pA parameter; in this way, $\mathrm{ETL}_{\mathrm{eff}}$ increased at the cost of ESP.

To obtain quantitative results, the PSF is calculated as the inverse Fourier Transform $\left(\mathrm{FT}^{-1}\right)$ of a vertical prof le of the image along the phase encoding direction (Fig. 2B, D), since the delta phantom can be interpreted as a delta input for the system. The FWHM is calculated by ftting the PSF to a Lorentzian function [20]. Blurring and signal enhancement are assessed by representing the maximum signal level and the FWHM of the PSF as a function of pA and $\mathrm{ETL}_{\mathrm{eff}}$. This experiment has not been performed in the scanner, as it requires complex procedures such as switching off the phase encoding gradients or deriving the PSF from a step phantom image.

Analytically, the $k$-space of the line phantom can be modeled as the convolution of two functions: a box-car function and an exponential given by $\exp \left(-\mathrm{TE} / \mathrm{T}_{2}\right)$, as it can be inferred from Fig. 2A. The $\mathrm{FT}^{-1}$ of this convolution is the product of a Lorentzian curve and a sinc function (Fig. 2B). Image blurring is proportional to the FWHM of the Lorentzian function, while ringing depends on the sinc function. In the case of the sHF-TSE acquisition (Fig. 2C), the $k$-space can be modeled as the sum of two truncated exponential functions convolved with a pulse, a model similar to the former one, though involving a more complex analytical calculation. However, the $k$-space of the threecircles phantom is not so easily modeled, thus making advisable the use of numerical simulation.

The three-circles phantom consists of three circles on a black background. Each circle has a gray level that simulates a different $\mathrm{T}_{2}: 30,62$ and $126 \mathrm{~ms}$, matching those used in the delta phantom. The series of sequences simulated for this phantom have the following main parameters: $\mathrm{TE}=$ $120 \mathrm{~ms}, \mathrm{TR}=2500 \mathrm{~ms}, \mathrm{pA}=100,80$ and $60 \%, \mathrm{ETL}_{\mathrm{eff}}=$ 15 echoes. The $\mathrm{T}_{2}$-related blurring produced by the sequence was obtained from a prof le along phase encoding direction, and measured as the width of the edge of each circle on the simulated image. Ringing was assessed by measuring background rippling on the same prof le. Simulated results for the three-circles phantom have been validated by comparison with the corresponding images acquired in the scanner (Fig. 5).

Finally, computer simulations were made using Matlab (Mathworks, Natick, MA, USA). The main steps [21,22] were as follows: two images (either true or synthetic) of the same object, corresponding to different TE and same TR (e.g., dual echo SE acquisition), are used to estimate the $T_{2}$ decay of each pixel, approximated by a monoexponential curve. This decay curve is used to calculate a simulated TSE echo train of the desired length. The echo time (TE) of each 
A
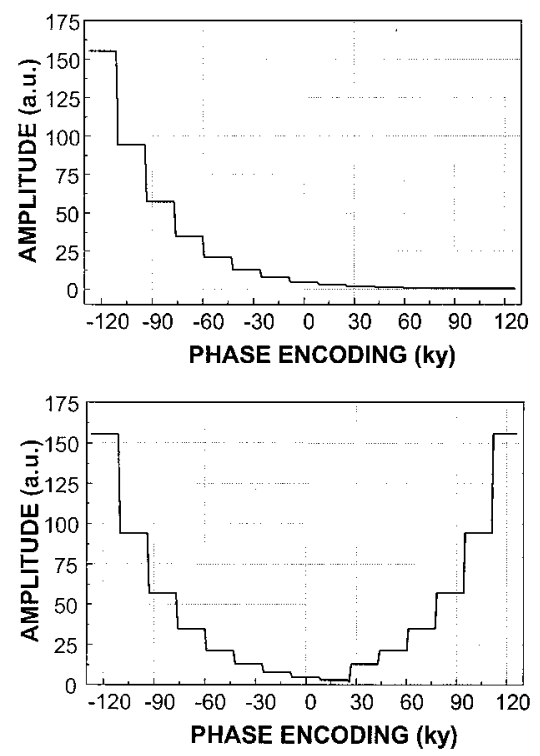

C
B
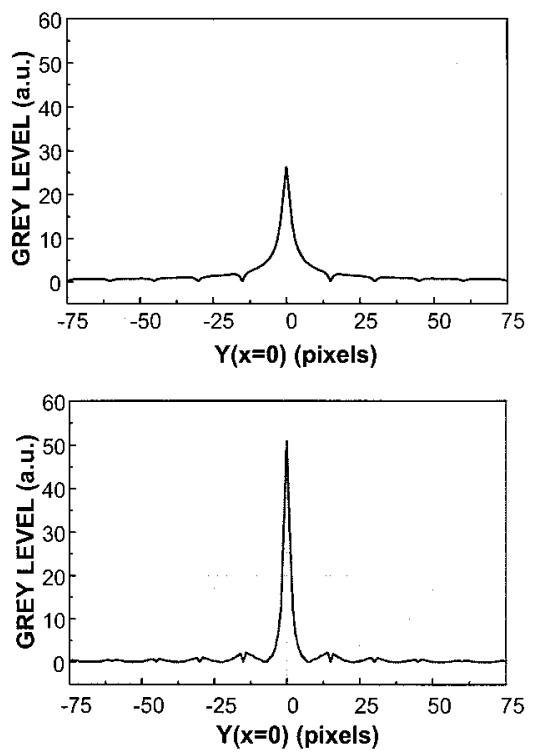

D

Fig. 2. $k$-Space weighting and PSF obtained for the synthetic one-line phantom in the case $\mathrm{T}_{2} / \mathrm{ETL} / \mathrm{ESP}=15 \mathrm{~ms} / 15 \mathrm{echoes} / 30 \mathrm{~ms}$. Plot $(\mathrm{A})$ represents the $k$-space for a full TSE acquisition and (B) its associated point spread function (PSF). Plot (C) represents the $k$-space for a HF-TSE acquisition with pA $=$ $60 \%$ and (D) its the corresponding PSF.

image is determined by both the echo spacing and $\mathrm{TE}_{\mathrm{eff}}$ selected by the user. The $k$-space of the simulated TSE image is built by pasting together bands taken from the Fourier transforms (FT) of each image of the train (Fig. 3). Each band corresponds to a segment of the fnal TSE $k$ space. At this step the program allows simulation of Half Fourier acquisition and the effect of noise (adding gaussian noise independently to the real and imaginary parts of the raw data). Finally, an inverse FT produces the resulting TSE/HF-TSE simulated image.

\subsection{Image acquisition}

True images have been used to assess the artifacts introduced by both the scanner and the biological system itself. The images were acquired using a 1.5 Tesla Gyroscan ACS II (Philips Medical Systems, The Netherlands). The sequences had the following main parameters: $\mathrm{TE}=120 \mathrm{~ms}$, $\mathrm{TR}=2500 \mathrm{~ms}, \mathrm{pA}=100,80$ and $60, \mathrm{ETL}_{\text {eff }}=15(\mathrm{ETL}$ $=15,12$ and 9 echoes), NEX $=2$, scan percentage $=$ $100 \%$, and acquisition time $=3 \mathrm{~min}$. Note that the ETL

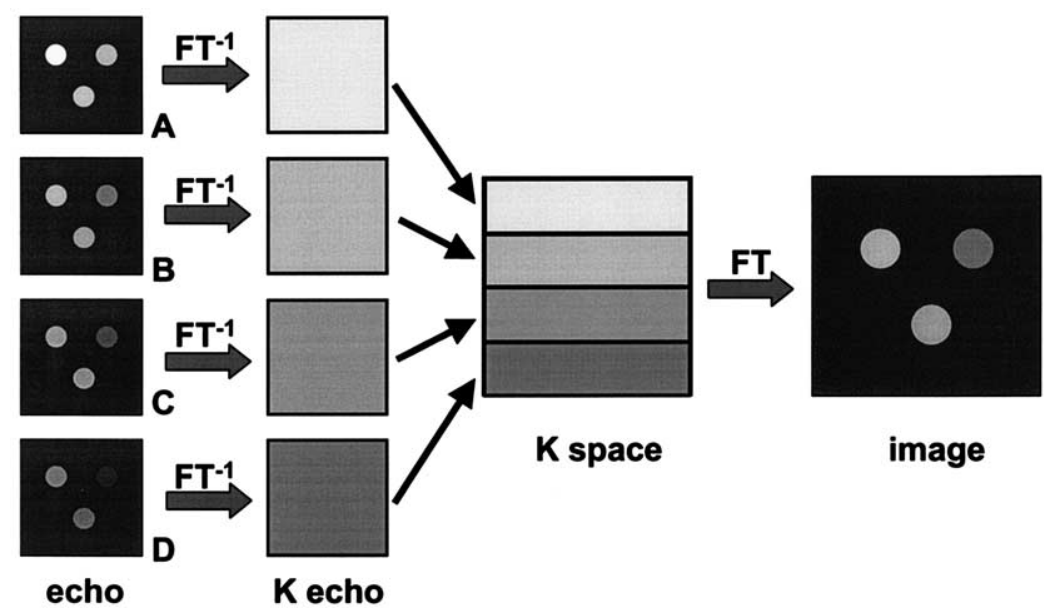

Fig. 3. Numerical simulation: The echo train in this example is formed from four images (A, B, C and D) of a phantom, each one weighted for a given TE. An inverse FT yields the raw data $(K$ echoes $)$ corresponding to the four "echoes." The $k$-space of the f nal image is made up of four segments $(\mathrm{ETL}=4)$, each one belonging to its own $K$ echo. 
changes according to the $\mathrm{pA}$ in order to keep $\mathrm{ETL}_{\mathrm{eff}}$ constant.

The phantom is made up of three cylindrical plastic tubes (Fig. 5) with a diameter of $4 \mathrm{~cm}$, flled with $40 \mathrm{cc}$ of a mixture of water and agar or water and agarose [5] in the following proportions:

Circle A: water and agar $1 \%$ by weight $\left(\mathrm{T}_{2}=139 \mathrm{~ms}\right.$, similar to tumor)

Circle B: water and agarose $2 \%$ by weight $\left(\mathrm{T}_{2}=58\right.$ ms, similar to fat)

Circle C: water and agarose $4 \%$ by weight $\left(\mathrm{T}_{2}=31\right.$ ms, similar to muscle).

$\mathrm{T}_{1}$ relaxation times of the phantoms are not relevant due to the TR used in the series of sequences.

The tubes were imaged in the coronal plane using a quadrature head coil, cranio-caudal phase encoding, f eldof-view $(\mathrm{FOV})=160 * 160 \mathrm{~mm}^{2}$ and slice thickness $=4$ $\mathrm{mm}$. Image blurring and ringing were assessed as in the previous case.

The visual effect on clinical images has been assessed on two anatomical locations: abdomen and knee. Five patients were examined for each location. Abdomen images were acquired in the axial plane with a quadrature body coil, anterior-posterior phase encoding direction, $\mathrm{FOV}=300 *$ $300 \mathrm{~mm}^{2}$ and slice thickness $=10 \mathrm{~mm}$. Knee images were acquired in the coronal plane with a quadrature knee coil, cranio-caudal phase encoding direction, FOV $=160 * 160$ $\mathrm{mm}^{2}$ and slice thickness $=4 \mathrm{~mm}$.

Patient images were analyzed with the same method as the phantom images: prof les obtained in the phase encoding direction provided information about blurring, ringing and signal level.

Signal-to-noise ratio (SNR) was calculated as the ratio between the average intensity in a uniform region of interest (ROI) within the signal region and the standard deviation of the background in a region free from artifacts [23].

\section{Results}

The sHF-TSE sequence proposed has been analyzed in terms of blurring, signal level and ringing, according to the percentage of acquired data and the echo train length used to generate or acquire the images, as explained in the previous section.

\subsection{Delta phantom}

\subsubsection{Results as a function of $p A$}

The percentage of acquired data determines both the image blurring and the signal level, represented, respectively, by the FWHM and the height of the PSF (Fig. 4). For lower values of pA (fewer data truly acquired) the maximum gray level increases and the FWHM decreases. The
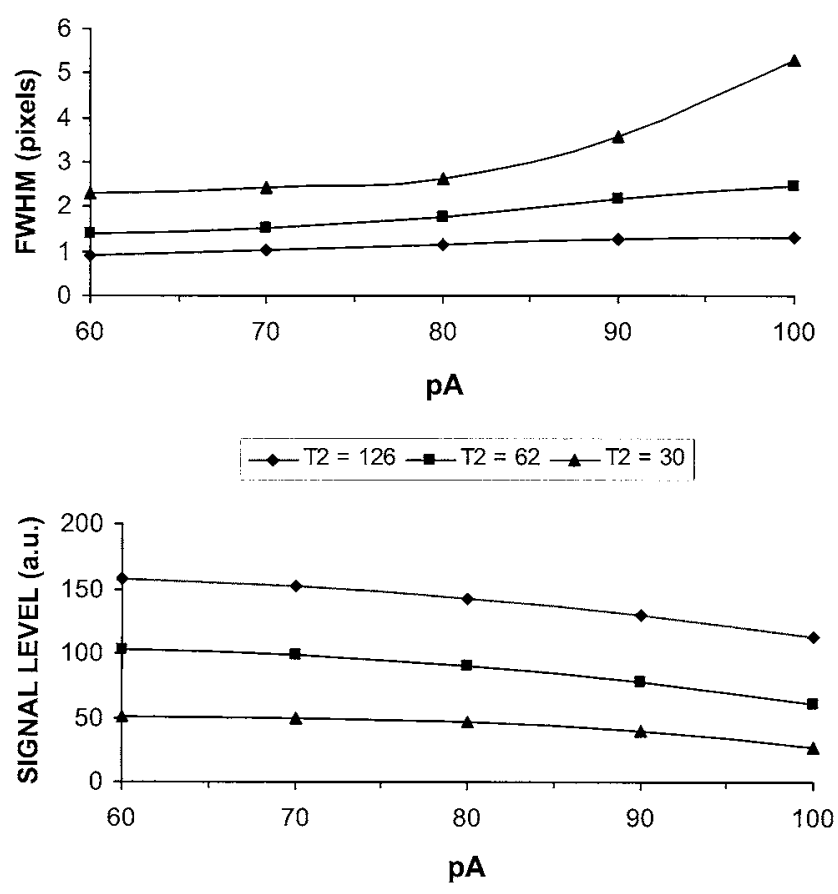

Fig. 4. Plot of FWHM and peak value of the PSF vs. pA for different T2 in the case $\mathrm{ETL}_{\text {eff }}=15$ echoes, $\mathrm{ESP}=15 \mathrm{~ms}$. The point $\left(\mathrm{pA}, \mathrm{T}_{2}\right)=$ $(100 \%, 30 \mathrm{~ms})$ ref ects the severe blurring for short $\mathrm{T}_{2} \mathrm{~s}$ introduced by the full TSE acquisition. The lower plate shows that the use of sHF-TSE sequence does not change the contrast of the TSE image.

most noticeable narrowing of the PSF is reached for the shortest $T_{2}$. For the longest $T_{2}(126 \mathrm{~ms})$, the enhancement in resolution is inconspicuous (less than one pixel) for the entire range of pA and ETL values tested, while at $T_{2}=30$ $\mathrm{ms}$ the narrowing of FWHM is larger than one pixel for every pA and ETL tested.

The lower panel of Fig. 4 shows the change of signal level according to $\mathrm{pA}$ : the signal level increases for lower values of $\mathrm{pA}$, following a similar pattern for the three $\mathrm{T}_{2}$ values tested. Therefore, no changes in image contrast are expected due to differences in $\mathrm{pA}$.

\subsubsection{Results as a function of ETL ${ }_{\text {eff }}$}

The change in FWHM due to the inf uence of $\mathrm{ETL}_{\text {eff }}$ is less than one pixel for all the $\mathrm{T}_{2} \mathrm{~s}$ and $\mathrm{pA}$ values used in the experiment. The signal level increases slightly as $\mathrm{ETL}_{\text {eff }}$ lengthen for all the $\mathrm{T}_{2} \mathrm{~s}$ tested.

\subsection{Three circles phantoms}

The images of the physical (Fig. 5) and synthetic phantoms have been analyzed by means of prof les in the phase encoding direction as described previously.

Figure 7 shows the prof les across circle $\mathrm{C}\left(\mathrm{T}_{2}=30 \mathrm{~ms}\right)$ for both the real and synthetic phantoms. The gray level value set for the circle $\mathrm{C}$ in the synthetic phantom is 5 


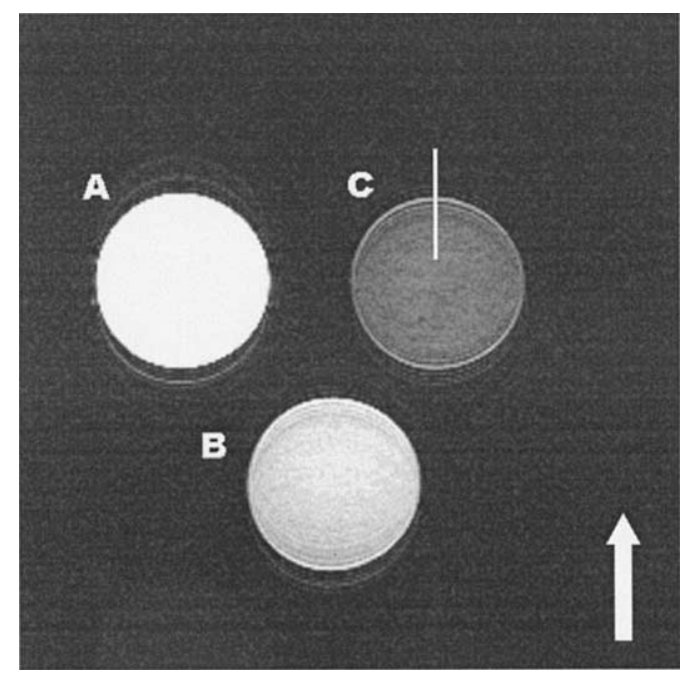

Fig. 5. Image of the three circles phantom at $\mathrm{pA}=60 \%$. The $\mathrm{T}_{2} \mathrm{~s}$ of the circles are $T_{2}(A)=139 \mathrm{~ms}, T_{2}(B)=31 \mathrm{~ms}$ and $T_{2}(C)=58 \mathrm{~ms}$. Note the conspicuousness of the ringing artifact. Phase encoding direction is vertical (white arrow).

arbitrary units while the background ${ }^{2}$ is set to 0 , in a 256 gray levels scale. Ideally, a prof le across the edge should be a step function, but it smooths under the inf uence of the TSE sequence. This blurring has been assessed on the simulated image by measuring the boundary width (in pixels), considering as background the $\mathrm{f}$ rst pixel with a value below 2.5 units. According to this criterion the boundary spans 6 pixels for $\mathrm{pA}=100,3$ pixels for $\mathrm{pA}=80$, and 4 pixels for $\mathrm{pA}=60$. The prof les on the true phantom are similar to the preceding ones: the boundary spans up to 8 pixels for $\mathrm{pA}=$ 100,5 for $\mathrm{pA}=80$, and 6 for $\mathrm{pA}=60$.

The Gibbs artifact becomes more conspicuous for lower percentages of $\mathrm{pA}$. The overenhancement of the edge visible on both panels of Fig. 6 is, in part, due to superposition of the blurred edge with the frst ring of the truncation artifact.

\subsection{Patient images}

Abdomen and knee locations imaged with sHF-TSE and TSE (Figs. 7,8) are qualitatively and quantitatively compared.

\subsubsection{Qualitative analysis}

A general edge enhancement is easily observed on the images acquired at $\mathrm{pA}=60 \%$ as compared with those at $\mathrm{pA}$ $=100 \%$. Small short- $\mathrm{T}_{2}$ structures are particularly enhanced, showing an increased contrast and higher signal level.

On the zoomed image of the abdomen shown in Fig. 7A, a slight hyperintense signal one-pixel wide is hardly visible in between the muscle $\left(T_{2} \sim 30 \mathrm{~ms}\right)$ of the anal sphincter

\footnotetext{
${ }^{2}$ The $\mathrm{T}_{2}$ value of $30 \mathrm{~ms}$ chosen for Circle $\mathrm{C}$ of the synthetic phantom yields a grey level of 5 units (in a 256 level grey scale) for $\mathrm{TE}=136 \mathrm{~ms}$.
}

$\mathrm{pA}=60$

physical phantom
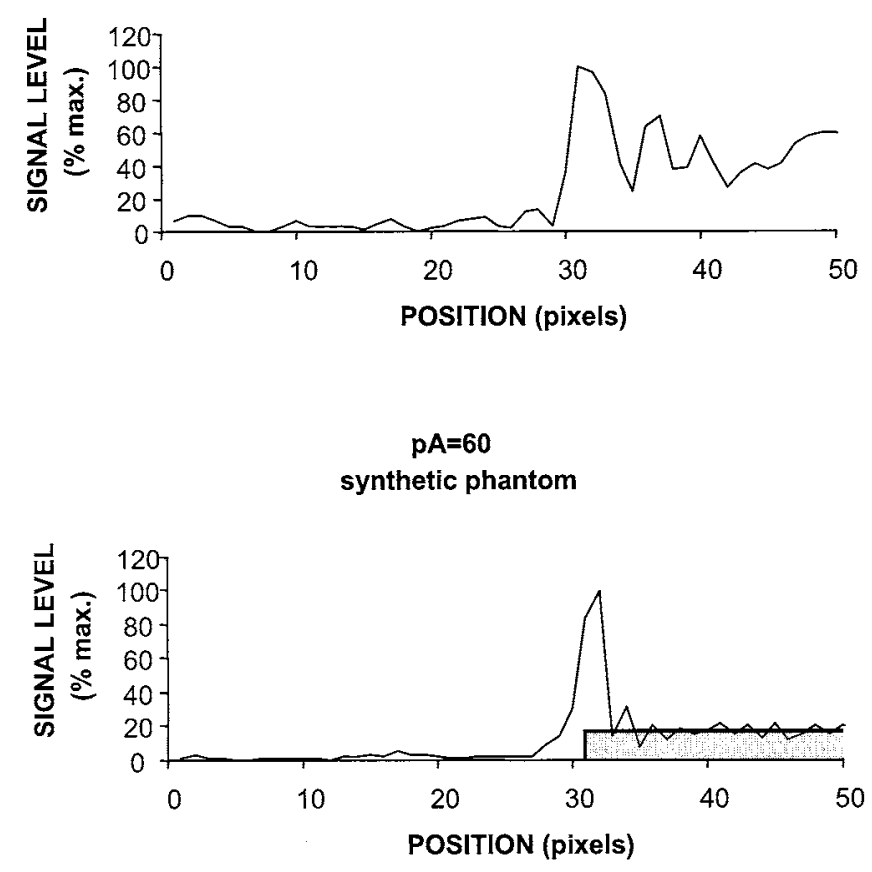

Fig. 6. Normalized prof les in the phase encoding direction across the B circle of the phantom in Fig. 5 (upper plate) and its equivalent synthetic phantom (lower plate), for the case $\mathrm{pA}=60 \%$. The grey rectangle superimposed on the synthetic phantom prof le corresponds to the prof le of the originally designed phantom.

for the $\mathrm{pA}=100$ acquisition. The zoomed image in Fig. 7B shows the enhancement of a hyperintense structure (liquid) that was overlapped by the muscle blurring in the case $\mathrm{pA}$ $=100 \%$.

The image of the knee presented in Fig. 8 shows several structures more clearly delineated for $\mathrm{pA}=60 \%$ than for $\mathrm{pA}=100 \%$. The cortical bone and the menisci look sharper on the HF image. The tissue that shows a higher enhancement is the cartilage located at both sides of the menisci, which have increased its gray level in approximately $25 \%$ and show a noticeably more discernible gap between them.

\subsubsection{Quantitative analysis}

Edge width and signal level were quantif ed on the pro$f$ les shown by each image. SNR were measured on the images as explained before. For the images of the abdomen, the hyperintense structure inside the muscle ring is represented in Fig. 7B as two equally high spikes with a gray level of approximately 900 arbitrary units. On the prof le of Fig. 7A only one spike is conspicuous with a gray level of roughly 800 . The width of the spikes remains the same for both cases. The prof le drawn across the cartilage shows a signal increasing of up to $50 \%$ (Fig. 8). Note the low signal value of the cortical bone, indicating the absence of blurring from adjacent tissues.

The paired images are shown with the same window 

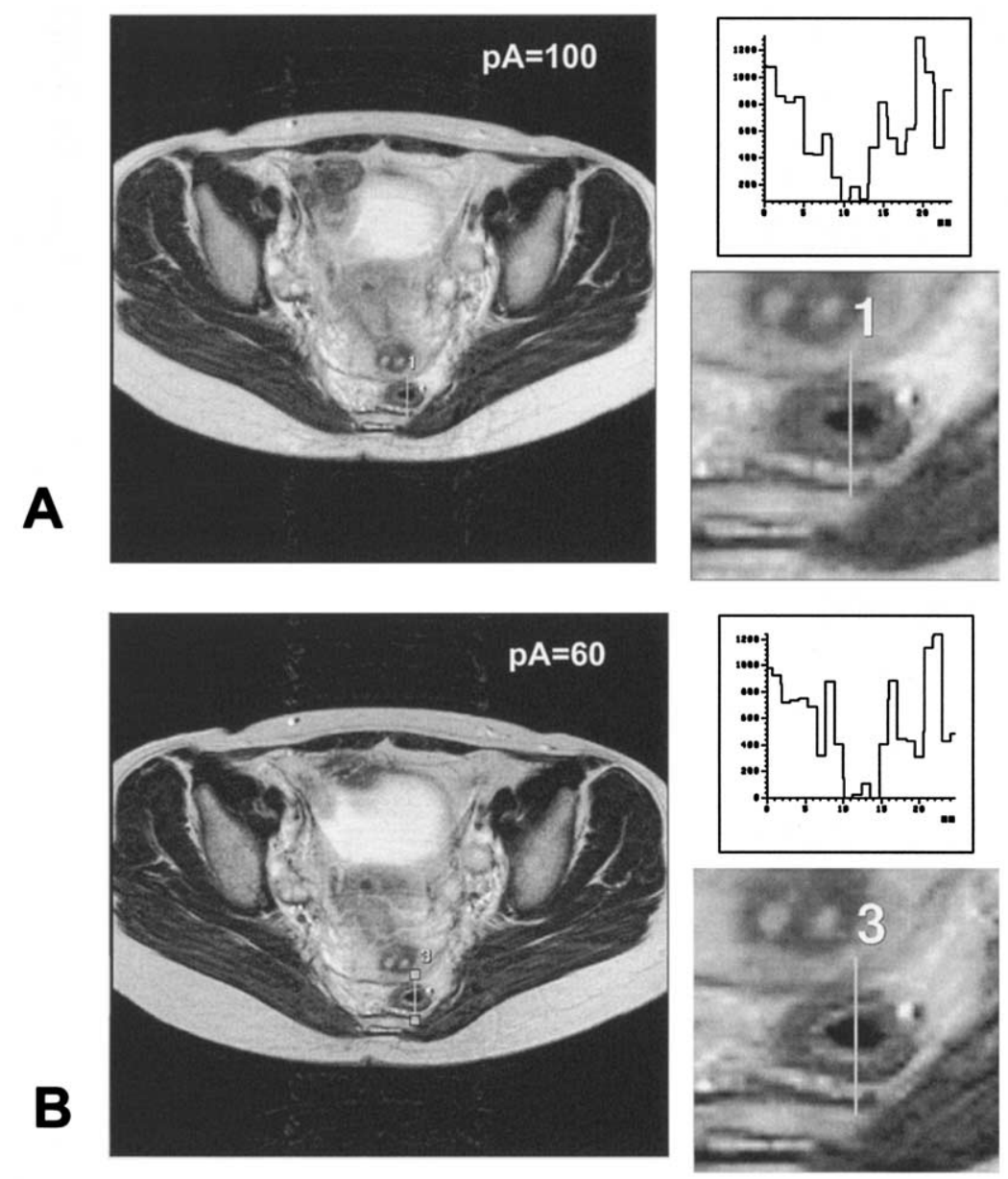

Fig. 7. A. Full acquisition image. The prof le along the line shown in the zoomed image reveals slightly hyperintense pixels inside the muscle ring. B. Image acquired at $\mathrm{pA}=60 \%$. The enhancement in f ne detail compared to the full acquisition image can be verif ed on the prof les shown. The ghosting artifact is more conspicuous in this case than at $\mathrm{pA}=100 \%$.

level and width. In the abdomen image, SNR decreases $17.6 \%$ for $\mathrm{pA}=80 \%$ and $31.7 \%$ for $\mathrm{pA}=60 \%$ with respect to the full acquisition, corresponding to a ratio of 1.47 between $\operatorname{SNR}(100)$ and $\operatorname{SNR}(60)$. In the knee images, the SNR decreases $18 \%$ for $\mathrm{pA}=80 \%$ and $30 \%$ for $\mathrm{pA}=60 \%$, yielding a ratio $\operatorname{SNR}(60) / \operatorname{SNR}(100)=1,45$. These f gures of SNR are in accordance with the theoretical prediction.

\section{Discussion}

The study of the combination of TSE sequences with Half Fourier techniques has been classically centered on the particular case known as HASTE (Half Fourier Acquisition Single Shot Turbo Spin Echo) $[24,25]$. The HASTE sequence $\mathrm{f}$ lls half of the $k$-space in one single shot, thus being a very fast sequence generally used to acquire relatively low resolution images, either for breath-hold abdominal imaging or for fast scanning of children or uncooperative patients.

This article proposes the use of a segmented multishot version of the HF-TSE sequence to achieve a partial recov- ery of the spatial resolution in $\mathrm{T}_{2}$-weighted TSE images. The single-shot case $(E T L=256)$ has been also included in our study for the sake of completeness, even though the goal of our work is not the study of the HASTE sequence, well characterized in previous works.

To our knowledge, the use of multishot HF-TSE sequences has only been described in [26], dividing the sequence into two shots, still with the main purpose of shortening acquisition time. As a difference with other previously proposed approaches, [9-12], the use of segmented HF-TSE does not require additional acquisitions nor increase acquisition time.

Several techniques have been proposed to reconstruct the $k$-space of HF acquisitions. Parametric models appear to provide a reliable estimation of the missing data [27] at the expense of computational complexity that prevents from providing real-time image reconstruction.

The enhancement in spatial resolution attained by our sequence is due to the improved symmetry of the echo train implicit in the sHF-TSE reconstruction. The decrease in blurring is more noticeable for short $\mathrm{T}_{2}$ structures, when the 


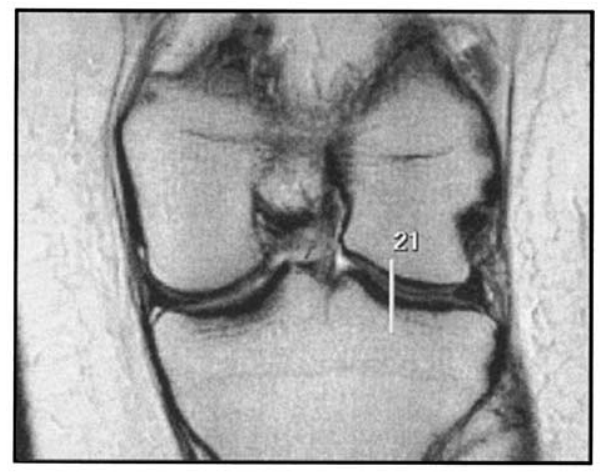

\section{$\mathrm{pA}=\mathbf{1 0 0}$}
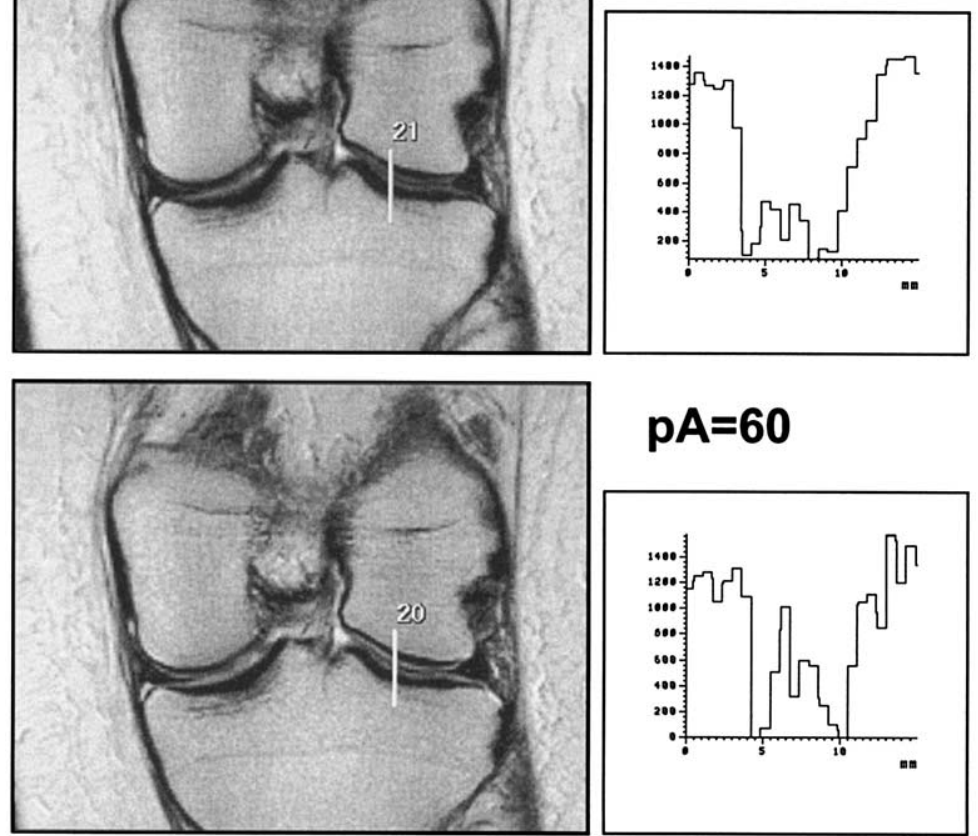

$\mathrm{pA}=60$

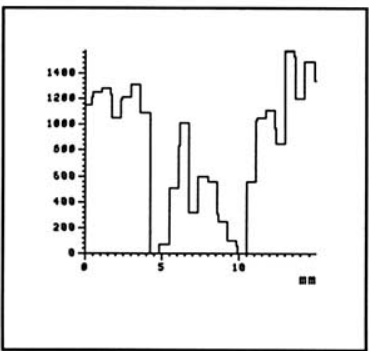

Fig. 8. The comparison between images shows better resolution and tissue contrast in the $\mathrm{pA}=60 \%$ case. The increased grey level of the cartilage and the sharper borders of the lower panel image are demonstrated on the adjacent prof les.

data acquisition time (proportional to the echo train length) is longer than the $\mathrm{T}_{2}$ of the tissue [28].

In our study, $\mathrm{ETL}_{\mathrm{eff}}$, TE and TR have been kept constant to preserve the image contrast along the whole series of sequences, then assuring that changes in contrast with respect to the reference image are due only to the HF acquisition. Being the TR $\mathrm{f}$ xed for every pA series, the acquisition time does not decrease, as could be expected. However, the multislice capacity of the sequence is increased, since a lower pA entails a shorter ETL that leaves free a longer section of the TR.

The most interesting features of the proposed sHF-TSE relate to the changes in spatial resolution as a function of $\mathrm{pA}$ and $\mathrm{ETL}_{\text {eff }}$. The resolution was measured as the FWHM of the PSF, which was obtained by software simulations and analytical validations when possible. The numerical simulation has proven to be rather valuable to characterize the sHF-TSE sequence, obtaining PSFs free from any other artifacts present in real acquisitions. Analytically, we found that the FWHM of the sHF-TSE sequence is no longer strictly proportional to $\mathrm{T}_{\text {eff }} * \mathrm{ESP} / \mathrm{T}_{2}$ (ESP $=$ echo spacing), as it is in conventional TSE acquisitions [2], though it is still inversely related to $\mathrm{T}_{2}$ (Fig. 4).

In full acquisition images (conventional TSE) short $\mathrm{T}_{2}$ tissues appear blurred, as expected [8]. Our results (Fig. 4) show that for short $\mathrm{T}_{2}$ values the choice of $\mathrm{pA} \leq 80 \%$ may produce images with better spatial resolution, narrowing the PSF up to half the original width for the full acquisition.

Our results also show that signal intensity increases with

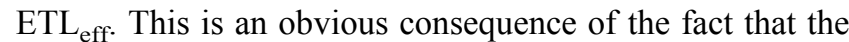
f rst echoes are acquired earlier when increasing $E T L_{\text {eff }}$, since the TE is kept constant. For instance, the frst echo in the case $\mathrm{ETL}_{\text {eff }}=9$ is acquired at a $\mathrm{TE}(1 \mathrm{st})$ of $24 \mathrm{~ms}$, while for a single shot acquisition TE(1st) is $0.9 \mathrm{~ms}$. As a consequence, the use of longer $\mathrm{ETL}_{\mathrm{eff}}$, and therefore a shorter ESP, in combination with HF, produces brighter images. There is also a signal increase as pA decreases, as expected. The signal intensity is given by the integral over the time domain signal. Neglecting noise considerations, the integral over a symmetrically complemented signal as in Fig. $2 \mathrm{C}$ is expected to be higher than the monotonously decaying function shown in Fig. 2A. This increase of signal level does not depend on the $T_{2}$ of the structure, thus not leading to changes in contrast.

However, lower pA values also convey a higher ringing artifact (Fig. 6), which may degrade image quality. According to our results from the synthetic phantom study, the $\mathrm{pA}$ $=80 \%$ value seems to achieve an optimum balance between signal intensity, edge resolution and ringing artifact.

Ringing artifact increases at lower pA due to the larger discontinuities between segments introduced in the "reconstructed" part of the $k$-space. For $\mathrm{pA}=100$ the $\mathrm{T}_{2}$ decay for the second half of the echo train is negligible, as shown in Fig. 2A. Figures $2 \mathrm{~A}$ and $2 \mathrm{C}$ allow us to compare the difference between the magnitude of the discontinuities for the TSE and for the HF-TSE acquisitions.

The ratio $\mathrm{SNR}_{\mathrm{SHF}-\mathrm{TSE}} / \mathrm{SNR}_{\mathrm{TSE}}$ measured on the patient images was in agreement with the theoretical prediction. In 
spite of the worse SNR provided by the SHF-TSE sequence, the increase in resolution of the images improved noticeably the recognition of small structures. A reasonable continuation for our work is the design of noise $\mathrm{f}$ lters adapted to the particular noise distribution of the sHF-TSE images, which could further improve the image quality.

On abdominal images, the sHF-TSE sequence led to an increased conspicuity of the respiration artifact, more intense at low pA values. This is reasonable since the movement-related phase errors are duplicated into the calculated part of the $k$-space, increasing their effect. Joint images, however, showed a noticeable enhancement in resolution at the expense of a small increase of the ringing artifact across sharp interfaces (bone-cortical bone), hardly visible on the images at bare eye.

\section{Conclusion}

The use of TSE secuences for the acquisition of heavily $\mathrm{T}_{2}$-weighted images produces a severe blurring of short- $\mathrm{T}_{2}$ small objects that became hardly conspicuous or may even disappear from the image. The segmented Half Fourier TSE (sHF-TSE) sequence proposed in this paper improves image quality, producing sharper $T_{2}$ images. Signif cant edge enhancement (up to $45 \%$ decrease in FWHM) with respect to a full acquisition has been achieved for $\mathrm{pA}=60 \%$ and $\mathrm{T}_{2}$ $=30 \mathrm{~ms}$ (equivalent to muscle). Different combinations of TR and ETL may lead to either a shorter aquisition time or an increased multislice capacity.

A negative aspect of sHF-TSE images is the lower SNR with respect to the standard TSE images, though the overall image quality seems still better because of the increase in resolution.

Another limitation of this sequence is the increased conspicuity of respiratory artifacts, typical in abdominal imaging. For this reason, the sequence seems particularly indicated for limb imaging, where periodic movement ghosts are not generally a problem. The best combination of parameters to achieve good resolution in $\mathrm{T}_{2}$-weighted images seems to be $\mathrm{pA}=80 \%$ with a relatively long $\mathrm{ETL}_{\text {eff }}$ (approximately 21 echoes)

\section{Acknowledgments}

This work was partially funded by grants FIS-00/36, Red Temática IM3 (FIS) and III-PRICIT (Comunidad de Madrid).

\section{References}

[1] Gatehouse PD, Bydder GM. Magnetic resonance imaging of short T2 components in tissue. Clin Radiol 2003;58:1-19.
[2] Listerud J, Einstein S, Outwater E, Kressel HY. First principles of Fast Spin Echo. Magn Reson Q 1992;4:199-244.

[3] Catasca J, Mirowitz S. T2-weighted MR imaging of the abdomen: fast spin-echo vs. conventional spin-echo sequences. AJR Am J Roentgenol 1994;162:61-7.

[4] Hennig J, Nauerth A, Friedburg H. RARE imaging: a fast imaging method for clinical MR. Magn Reson Med 1986;3:823-33.

[5] Mitchell D, Outwater E, Vinitski S. Hybrid RARE: implementations for abdominal MR imaging. J Magn Reson Imaging 1994;4: $109-17$.

[6] Atlas S, Hackney D, Listerud J. Fast spin-echo imaging of the brain and spine. Magn Reson Q 1993;9:61-83.

[7] Fellner C, Fellner F, Schmitt R, Helmberger T, Obletter N, BöhmJurkovic H. Turbo spin-echo sequences in magnetic resonance imaging of the brain: physics and applications. MAGMA 1994;2:51-9.

[8] Constable RT, Gore JC. The loss of small object contrast in variable TE imaging: implications for FSE, RARE and EPI. Magn Res Med 1992;28:9-24.

[9] Mulkern RV, Wong STS, Jakab P, Bleier AR, Sandor T, Jolesz FA. CPMG Imaging sequences for high feld in vivo transverse relaxation studies. Magn Res Med 1990;16:67-79.

[10] Keller P, Heiserman J, Fram E, Rand S, Drayer B. A Nyquist modulated echo-to-view mapping scheme for fast spin-echo imaging. Magn Res Med 1995;33:838-42.

[11] Zhou X, Liang ZP, Cofer GP, Beaulieu CF, Suddarth SA, Johnson GA. Reduction of ringing and blurring artifacts in fast spin-echo imaging. J Magn Reson Imaging 1993;3:803-7.

[12] Hennig J. Multiecho imaging sequences with low refocusing fip angles. J Magn Reson 1988;78:397-407.

[13] Feinberg DA, Hale JD, Watts JC, Kaufman L, Mark A. Halving MR imaging time by conjugation: demonstration at $3.5 \mathrm{kG}$. Radiology 1986;161:527-31.

[14] Vlaardingerbroek MT, den Boer JA. Magnetic resonance imaging. Berlin: Springer, 1996.

[15] Constable RT, Anderson AW, Zhong J, Gore JC. Factors inf uencing contrast in Fast Spin Echo MR Imaging. Magn Reson Imaging 1992; 10:497-511.

[16] Wild JM, Paley MN, Viallon M, Schreiber WG, van Beek EJ, Grif$\mathrm{f}$ ths PD. k-space fltering in 2D gradient-echo breath-hold hyperpolarized 3He MRI: spatial resolution and signal-to-noise ratio considerations. Magn Reson Med 2002;47:687-95.

[17] Partain CL, Price R, Patton J, Kulkarni M, James AE. Magnetic resonance imaging. Philadelphia: W. B. Saunders Company, 1988.

[18] Abe Y, Yamashita Y, Tang Y, Namimoto T, Takahashi M. Calculation of $\mathrm{T} 2$ relaxation time from ultrafast single shot sequences for differentiation of liver tumors: comparison of echo-planar, HASTE, and spin-echo sequences. Radiat Med 2000;18:7-14.

[19] Chan YL, Lee SF, Yu SC, Lai P, Ching AS. Hepatic malignant tumour versus cavernous haemangioma: differentiation on multiple breath-hold turbo spin-echo MRI sequences with different T2-weighting and T2-relaxation time measurements on a single slice multi-echo sequence. Clin Radiol 2002;57:250-7.

[20] Papoulis A. Circuits and systems: a modern approach. New York: Holt, Rinehart and Winston, 1980.

[21] Lemke HU, Vannier MW, Inamura K, Farman A, editors. CAR '96 computer assisted radiology. Paris: Elsevier, 1996:995.

[22] Desco M, Santa Marta C, Lafuente J, Vaquero JJ, del Pozo F, García-Barreno P. Software simulation of a partial K-space image with a FSE sequence. Radiology 1996;201:152.

[23] Kaufman L, Kramer D, Crooks L, Ortendahl D. Measuring signal to noise ratios in MR imaging. Radiology 1989;173:265-7.

[24] Miyazaki T, Yamashita Y, Tsuchigame T, Yamamoto H, Urata J, Takahashi M. MR cholangiopancreatography using HASTE (halfFourier acquisition single-shot turbo spin-echo) sequences. AJR Am J Roentgenol 1996;166:1297-303.

[25] Miller S, Schick F, Scheule AM, Vogel U, Hiller R, Strotmann C, Naegele T, Hahn U, Claussen CD. Conventional high resolution 
versus fast $\mathrm{T}(2)$-weighted MR imaging of the heart: assessment of reperfusion induced myocardial injury in an animal model. Magn Reson Imaging 2000;18:1069-77.

[26] Sugahara T, Korogi Y, Hirai T, et al. Comparison of HASTE and segmented-HASTE sequences with a T2-weighted fast spin-echo sequence in the screening evaluation of the brain. AJR Am J Roentgenol 1997;169:1401-10.

[27] McColl RW, Scarlata MA. Partial Fourier MRI: AR models with SVD. IEEE Nuc Sci Symp Med Imag Conf 1999;74:M7-110.

[28] Hennig J. K-space sampling strategies. Eur Radiol 1999;9:1020-31. 